\section{LA FORMACIÓN EN LOS COLEGIOS PROFESIONALES, EL APRENDIZAJE A LO LARGO DE LA VIDA, LA ACREDITACIÓN PROFESIONAL Y LA CALIDAD DE SERVICIOS A LOS USUARIOS}

\author{
Fernando Chacón Fuertes \\ Decano del Colegio Oficial de Psicólogos de Madrid \\ Vicesecretario de la Unión Interprofesional \\ de la Comunidad de Madrid
}

\section{THE FORMATION IN PROFESSIONAL ASSOCIATIONS, THE LIFELONG EDUCATION, THE PROFESSIONAL AUTHORIZATION AND THE QUALITY OF SERVICES AIMED AT USERS}

\begin{abstract}
The main aims of the Professional Associations, which are ruled by the public law, are the organization of the practice of the diverse professions, the exclusive representation and the defense of professional interests. The social change has boosted three different aspects: the quality of the service, the needed learning "during the whole professional life" and the certification. There are diverse examples of professional accreditation: the court-appointed counsels, the EUROPSY and the European certificate for all EFPA's Experts on Psychotherapy. The contents and the phases of the curriculum are described, the continuous professional development (CPD) and the Certificate for the accreditation of the Psychologists specialists on psychotherapy.
\end{abstract}

KEY WORDS: Professional associations, nature; function; services' quality; continuous education and training; professional credentials; Euro-Psy, Psychology European Certificate; curriculum; Certificate for the accreditation of the Psychologists experts on psychotherapy.

\section{1. ¿Qué son los Colegios Profesionales?}

A pesar de que algunos Colegios Profesionales surgieron hace más de trescientos años directamente relacionados con los antiguos gremios, existe un gran desconocimiento sobre sus funciones y naturaleza jurídica, por eso aunque a algún lector pueda parecerle innecesario, creo oportuno delimitar lo que es un Colegio Profesional de acuerdo con el derecho administrativo español, y sus diferencias con las asociaciones, así como sus diferencias y semejanzas con otras instituciones profesionales internacionales.

La Ley 2/1974, de 13 de febrero, sobre Colegios Profesionales establece en su artículo 1.1. que:

"Los Colegios Profesionales son Corporaciones de derecho público, amparadas por la Ley y reconocidas por el Estado,
RESUMEN: Los Colegios profesionales, corporaciones de derecho público, tienen como fines esenciales la ordenación del ejercicio de las profesiones, la representación exclusiva de las mismas, y la defensa de los intereses profesionales. El cambio social ha impulsado tres aspectos: la calidad del servicio, el necesario aprendizaje "a lo largo de la vida profesional" y su certificación. Ejemplos de acreditación profesional son los Abogados del Turno de Oficio, el EURO-PSY y el Certificado Europeo de Experto en Psicoterapia de la EFPA. Se describen el contenido y las fases del currículum, el Desarrollo Profesional Continuo (DPC) y el Certificado de Acreditación de Psicólogos Especialistas en Psicoterapia.

PALABRAS CLAVE: Colegios Profesionales, naturaleza; función; calidad de servicios; formación contínua; acreditación profesional; Euro-Psy, certificado europeo en Psicología; currículum; certificado de acreditación de psicólogos especialistas en psicoterapia.

con personalidad jurídica propia y plena capacidad para el cumplimiento de sus fines."

El Tribunal Constitucional definió los Colegios Profesionales en su sentencia 20/1988, de 18 de febrero, como:

"Los Colegios Profesionales son corporaciones sectoriales que se constituyen para defender primordialmente los intereses privados de sus miembros pero que también atienden a finalidades de interés público, en razón de las cuales se configuran legalmente como personas jurídico-públicas o Corporaciones de Derecho Público cuyo origen, organización y funciones no dependen sólo de la voluntad de sus asociados, sino también, y en primer término, de las determinaciones obligatorias del propio legislador, el cual, por lo general, les atribuye asimismo el ejercicio de funciones propias de las Administraciones territoriales o permite a 
estas últimas recabar la colaboración de aquéllas mediante colaboraciones expresas de competencias administrativas, lo que sitúa a tales Corporaciones bajo la dependencia o tutela de las citadas Administraciones territoriales titulares de las funciones o competencias ejercidas por aquéllas".

Este texto deja bien a las claras las dos vertientes de los Colegios Profesionales. En primer lugar, surgen por la voluntad asociativa de sus miembros; pero tienen además funciones de interés general "delegadas" por la Administración, por lo que se constituyen como Corporaciones de Derecho Público. Este "interés general" que justificaría la creación de los colegios y la obligatoriedad de la colegiación está explícitamente puesto de manifiesto en la siguiente inscripción que se encuentra en la sede del Colegio de Médicos de Madrid que recoge un texto de sus normas constituyentes:

"Por orden 1796 se fundó el primer Colegio de Médicos de Madrid, con el nombre de Real Colegio de Medicina para que '... hallen pronto y seguro socorro los hombres oprimidos de la enfermedad, angustia y dolor'. Dándose la atribución de habilitar a los Médicos para ejercer la Medicina y sancionar a los 'Profesionales del Arte de Curar que se excedan de sus debidos límites, no permitiendo de ningún modo los Curanderos'".

Por tanto, es evidente que ése es el sentido original de la creación de los Colegios. Cosa distinta es que parte de la sociedad considera que esta finalidad ha sido desvirtuada.

La función más importante "delegada" por la Administración en los Colegios es sin duda el ordenamiento de la profesión, específicamente el control deontológico, que incluye el poder sancionador. Es decir, los Colegios Profesionales son asociaciones y por ello, defienden y representan a la profesión, pero son corporaciones de derecho público porque esa finalidad está supeditada al interés general y de los usuarios de los servicios profesionales en particular'.

Sin duda, es este doble carácter lo que determina la singularidad del ordenamiento jurídico de lo Colegios Profesionales en España.

¿Cuáles son las diferencias, entonces, entre un Colegio Profesional y una Asociación Profesional? El siguiente cuadro pueda ayudarnos a sintetizarlo:

\begin{tabular}{|l|l|}
\hline ASOCIACIÓN PROFESIONAL & COLEGIO PROFESIONAL \\
\hline Libertad de creación & Constitución por ley o decreto-ley \\
\hline Naturaleza privada & Corporación de Derecho Público \\
\hline Asociación voluntaria & Adscripción obligatoria ${ }^{2}$ \\
\hline Ausencia de representatividad exclusiva & Representatividad exclusiva \\
\hline Reconocimiento por su porcentaje de representatividad legal & Reconocidos como Corporación de Derecho Público \\
\hline $\begin{array}{l}\text { No imprescindible cualificación profesional profesional (salvo que una } \\
\text { Asociación lo establezca) }\end{array}$ & Cualificación profesional obligatoria \\
\hline $\begin{array}{l}\text { Ejercicio de la profesión sin título obligatorio y voluntariedad de } \\
\text { asociacionismo }\end{array}$ & $\begin{array}{l}\text { Ejercicio de la profesión con habilitación y regulación según los } \\
\text { Estatutos del Colegio }\end{array}$ \\
\hline Organización y representación de intereses privados & Organización y representación de intereses públicos y privados \\
\hline
\end{tabular}

¿Cómo son las organizaciones profesionales en otros países occidentales? En países como Italia (las denominadas "Ordines") o Portugal la naturaleza jurídica de las corporaciones profesionales es muy similar a la española. En otros paises como los de origen anglosajón es muy distinta, ya que hablamos de entidades de adscripción voluntaria.
Pero en realidad, esta diferencia es más aparente que real, ya que tienen delegadas por su administración el reconocimiento de la cualificación profesional; y así se recoge incluso en la normativa europea sobre esta temática. El Real Decreto 1837/2008, de 8 de noviembre, por el que se incorporan al ordenamiento jurídico español la Directiva 
2005/36/CE, del Parlamento Europeo y del Consejo, de 7 de septiembre de 2005, y la Directiva 2006/100/CE, del Consejo, de 20 de noviembre de 2006, relativas al reconocimiento de cualificaciones profesionales dice:

"3. Igualmente, quedará equiparada a una profesión regulada,... la profesión ejercida por los miembros de una asociación u organización de las que se mencionan en el anexo I. Se trata de entidades que gozan de un reconocimiento especial por parte de un Estado miembro de la Unión Europea, que expiden a sus miembros un título de formación, garantizando que cumplen normas profesionales dictadas por ellas y les otorgan el derecho a utilizar profesionalmente un diploma, una abreviatura o un rango correspondiente a dicho título de formación" (art. 4.3.).

Es decir, para ser reconocido como profesional basta con la acreditación de estas asociaciones, cumpliendo los requisitos establecidos por ellas. En el mencionado anexo I se lista una serie de asociaciones irlandesas y británicas entre las que podemos encontrar la Chartered Society of Physiotherapy, la Royal Society of Chemistry, la British Psychological Society, o la Institution of Civil Engineers.

Creame el lector si le digo que muchos colegios profesionales españoles se cambiarían sin dudar por estas asociaciones profesionales británicas, porque sus atribuciones además del control de la profesión, les permite influir en qué tipo de formación da acceso a la misma.

En los Estados Unidos muchos estados, en algunas profesiones, como la abogacia, delegan en las asociaciones profesionales de teórica adscripción voluntaria, la organización y ejecución de los exámenes que permiten el ejercicio profesional en dicho Estado.

\section{LAS FUNCIONES DE LOS COLEGIOS PROFESIONALES}

La Ley 2/1974, de 13 de febrero, sobre Colegios Profesionales dispone:

"Son fines esenciales de estas Corporaciones la ordenación del ejercicio de las profesiones, la representación exclusiva de las mismas y la defensa de los intereses profesionales de los colegiados". y entre las funciones que se recogen en el artículo 5 se encuentran:

"e) Estar representados en los Patronatos Universitarios.

f) Participar en la elaboración de los planes de estudio e informar las normas de organización de los Centros docentes correspondientes a las profesiones respectivas y mantener permanente contacto con los mismos y preparar la información necesaria para facilitar el acceso a la vida profesional de los nuevos profesionales...

j) Organizar actividades y servicios comunes de interés para los colegiados, de carácter profesional, formativo, cultural, asistencial y de previsión y otros análogos, proveyendo al sostenimiento económico mediante los medios necesarios... r) Organizar, en su caso, cursos para la formación profesional de los postgraduados."

Como podemos comprobar, y a pesar de que esta norma tiene más de treinta y cinco años de vigencia y está centrada en las funciones corporativistas, ya establecía claramente el papel de los Colegios tanto en la formación de pregrado, como de posgrado.

Cabe destacar que, aunque sin mencionar explícitamente el concepto calidad de los servicios profesionales, de entre las muchas funciones propias de los colegios profesionales, las recogidas en la ley están orientadas precisamente a ese fin: la función de ordenación de la actividad profesional, velando por la ética profesional, por el respeto debido a los derechos particulares y ejerciendo la facultad disciplinaria sobre los profesionales colegiados; u otras funciones como la adopción de medidas para evitar el intrusismo profesional, el visado de trabajos profesionales o la organización de actividades de carácter formativo para los profesionales colegiados, en pro de mejorar la calidad de los servicios prestados por éstos, que al final redundarán en los consumidores y usuarios de los mismos.

Al amparo de esta normativa, los Colegios Profesionales españoles han desarrollado importantes programas formativos de posgrado, algunos de ellos muy ambiciosos $^{3}$ incorporando las NTI, dirigidos principalmente a sus afiliados, aunque en la mayoría de los casos también están abiertos a estudiantes de los últimos cursos para facilitar el acceso de éstos a la vida laboral en diversos ámbitos. 
Sin embargo, el desarrollo de las funciones e) y f) ha sido más irregular dependiendo de las relaciones que cada Colegio mantuviese con sus respectivas Facultades o Escuelas Universitarias. Así, los Colegios no están representados en los órganos que han sustituido a los Patronatos Universitarios; y en la mayoría de los casos su participación en la elaboración de los planes de estudio es más bien escasa, y se limita a emitir un informe valorativo del mismo. Incluso esta función prevista en la Ley de Colegios Profesionales se ha visto limitada en el Real Decreto 1393/2007, de 29 de octubre, de ordenación de las enseñanzas universitarias oficiales, en cuyo ANEXO I sobre la Memoria para la solicitud de verificación de Títulos Oficiales en su punto 2.2 establece que los informes de los Colegios Profesionales, en igualdad de condiciones que las asociaciones, pueden ser referentes externos a la Universidad proponente que avalen la adecuación de la propuesta a criterios nacionales o internacionales. Es decir, ni siquiera se considera requisito obligatorio para verificar un título el contar con el informe del Colegio, con independencia de que éste sea positivo o no.

\section{Calidad de Servicios, Formación Continua y ACreditación Profesional}

Sin duda, mucho ha recorrido y cambiado la sociedad española es estos treinta y cinco años. Cambios que para lo que nos ocupa, pueden resumirse en tres aspectos: la revolución de la cultura de la "calidad de servicio", la necesidad del "aprender a lo largo de la vida", y el cambio de perspectiva de los fines de los Colegios desde la finalidad meramente corporativa al interés social general.

Cuando se publicó la vigente Ley de Colegios Profesionales en 1974 apenas había empezado a utilizarse el concepto "calidad de servicio" en el mundo; por lo que era absolutamente impensable que esta norma no recogiera ninguna alusión al mismo. Es este tiempo, la calidad se ha hecho omnipresente al hablar tanto de producción de bienes como de servicios. No me refiero tanto a si efectivamente se prestan o producen los servicios y/o bienes con la calidad suficiente, me refiero más bien a un cambio cultural. La calidad se ha introducido como un valor inobjetable en nuestra sociedad. Prestar servicios de calidad es el fin último de profesionales y empresas. Crear sistemas y procedimientos que favorezcan la calidad de los servicios profesionales a los usuarios, consumidores, clientes o paciente es actualmente una de las finalidades principales de la Administración.

El "aprender a lo largo de la vida" no es sólo un derecho reconocido por la UNESCO ${ }^{4}$; sino que se trata de una necesidad. Hasta el último siglo la humanidad necesitaba centenares de años para duplicar sus conocimientos e información; actualmente le basta con apenas dos décadas. Si ha principios del siglo $X X$, todavía podría ser imaginable un médico o un ingeniero que desarrollara su labor durante veinticinco años o más sin apenas formación después de su licenciatura, actualmente es completamente impensable. Por tanto, los profesionales requieren una actualización permanente si se pretende que presten sus servicios con los niveles de calidad exigible. Éste es, sin duda, el principal cambio habido en la formación de los profesionales en las últimas décadas, y se enlaza directamente con el cambio de perspectiva en la función de los Colegios Profesionales. Los Colegios Profesionales son tales y no meras asociaciones, porque obedecen a intereses generales y no sólo corporativos, como dijimos anteriormente. Eso es asi desde su origen, aunque no es menos cierto que desde hace ya demasiados años muchas entidades colegiales han dedicado más tiempo y esfuerzo a promover los intereses particulares frente a los sociales. Pero, en los últimos años se ha

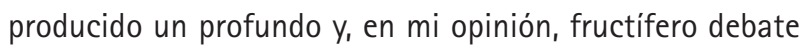
dentro de los Colegios Profesionales sobre su función social. Tómese como ejemplo, las recientes declaraciones del presidente de Unión Profesional de Valencia D. Francisco Real, en unas recientes declaraciones a la publicación electrónica Diario Crítico de la Comunidad Valenciana: "Ésa es la principal función de los colegios profesionales: mejorar la calidad del profesional para mejorar la calidad del servicio al ciudadano".

Este cambio de perspectiva está siendo recogido por la Administración y así en el Anteproyecto de Ley de Modificación de diversas leyes para su adaptación a la Ley....... sobre el libre acceso a las actividades de servicios y su ejercicio, en el que se prevé una modificación de la actual Ley de Colegios Profesionales, se redefinen los fines de estas corporaciones de la siguiente manera:

"3. Son fines esenciales de estas Corporaciones la ordenación del ejercicio de las profesiones, la representación 
exclusiva de las mismas, la defensa de los intereses profesionales de los colegiados y la protección de los intereses de los consumidores y usuarios de los servicios de sus colegiados..." (art. 5.1).

Es decir, si el Anteproyecto queda aprobado con su actual redacción se consagrará no sólo como función, sino como fin esencial de los Colegios la defensa de los intereses de los usuarios de los servicios profesionales.

Por tanto, la formación que facilitan los Colegios Profesionales a sus colegiados no son un mero servicio a los colegiados, sino que deben entenderse dentro de un sistema de mejora de la calidad de los servicios profesionales. Ahora bien, para que ese sistema sea completo, es preciso que certifique que el profesional ha participado en actividades de formación continua; o que acredite que se ha especializado en un determinado ámbito, técnica, procedimiento, etc.

Algunos liberales económicos a ultranza piensan que no debe existir ninguna entidad acreditadora, y será el propio mercado "libre" quien discrimine al final entre los profesionales que prestan servicios de calidad y los que no. Otros, entre los que me encuentro, consideramos que no está de más que alguna entidad envié una señal al mercado, mejor, a la sociedad, sobre si un determinado profesional cumple unos determinados requisitos o no. Esta entidad, sin duda debe ser la Administración, o en su defecto los Colegios como corporaciones de derecho público.

La Administración tiene sistema de certificación de la formación continua de los profesionales en dos ámbitos como son en el educativo y el sanitario, donde las administraciones son los principales empleadores. El del ámbito sanitario se denomina Sistema de Acreditación de Formación Continuada del Sistema Nacional de Salud, y acredita la formación que después será valorada por las administraciones en todos los procesos de selección, promoción y desarrollo de carrera profesional. Algo similar ocurre con los cursos acreditados en el sistema educativo, y ambos procesos de acreditación son de gran utilidad en los ámbitos de la función pública; pero su aplicación en el ámbito de los servicios privados es muy escasa. Por otra parte, debido a su coste, un sistema de acreditación de formación continua dependiente de la Administración que abarque a todas las profesiones $y$, en especial, el ejercicio liberal de las mismas, es impensable. Nadie mejor que los colegios, conocedores de la especificidad de la profesión, para crear sellos de calidad. Los sellos o marcas de calidad certifican que se cumple con determinadas normas. Con ellos, se está certificando que los servicios a los que se les concede, son objeto de las evaluaciones y controles que se establecen en los sistemas de certificación. Para ello los colegios habrán de identificar aquellos aspectos de su profesión que pueden resultar evaluables; así como establecer normas o protocolos que deberán cumplir los colegiados en la prestación de los servicios y servirán para la evaluación de la calidad de los mismos. Del mismo modo, habrán de implantar departamentos o servicios colegiales de certificación de calidad para la evaluación del cumplimiento de las normas y la comprobación del sistema de calidad, para la prestación del servicio profesional y para la inspección del servicio profesional ${ }^{5}$. El resultado final del proceso de certificación será la obtención por el profesional colegiado o por la sociedad profesional del certificado por el que se declarará la conformidad, en caso de que así fuese, del servicio profesional evaluado y la concesión del derecho de uso de la marca correspondiente que, a partir de ese momento, podrá utilizarse en los servicios certificados.

Todo ese proceso de certificación diferenciará a los profesionales que, en determinados ámbitos, prestan sus servicios con la calidad garantizada por el colegio de aquellos que no se hayan sometido a tal evaluación. Esto redundará en una mejora de la información de los clientes, pacientes o usuarios de los servicios profesionales y de la calidad general de los servicios profesionales ofrecida en el mercado.

Por último, existe otro ámbito importante a destacar, el de acreditación de personas, esto es, el reconocimiento formal de la cualificación de un profesional para poder realizar una actividad o conjunto de actividades o poder ocupar un puesto específico dentro de una organización (perfil profesional), puede jugar un papel fundamental como referente no regulado.

La entidad acreditada o entidad de certificación es el garante de la objetividad e imparcialidad de este proceso de reconocimiento formal de la cualificación del profesional, por lo que debe contar con estructuras en las que estén representadas todas las partes interesadas en el perfil profesional especifico; y es aquí donde surge la oportunidad 
para los colegios profesionales, ya que en ellos están integrados todos los profesionales y, por tanto, son menos susceptibles de introducir sesgos y están sometidos al control democrático de sus miembros.

En esta línea, desde la Unión Interprofesional de la Comunidad de Madrid, Asociación de Colegios Profesionales de Madrid, se viene trabajando desde hace años, a través de las Comisiones de Calidad y de Certificación y Cualificación Profesional, facilitando a los colegios y a sus colegiados una oportunidad para la mejora y el progreso a nivel institucional, profesional y personal.

Veamos algunos ejemplos de certificación y acreditación de la formación.

\section{EJEMPLOS DE ACREDITACIÓN PROFESIONAL. Los abogados del Turno de Oficio. El EURO-PSY y el Certificado Europeo de Experto en Psicoterapia de la EFPA ${ }^{6}$}

\subsection{Los abogados del Turno de Oficio}

Un buen ejemplo de exigencia de formación acreditada para acceder a prestar determinados servicios profesionales lo constituye el régimen de acceso al turno de oficio del Colegio de Abogados. Estos requisitos fueron inicialmente exclusivamente colegiales, y posteriormente fueron reconocidos normativamente por la Administración. Para ejercer la profesión de abogado, hasta que no entre en vigor la Ley 34/2006, de 30 de octubre, sobre el acceso a las profesiones de Abogado y Procurador de los Tribunales el 30 de octubre de 2011, es suficiente acreditar la titulación de licenciado en Derecho y la incorporación a un colegio profesional, no se necesita cumplir ningún requisito de formación posterior a la licenciatura, ni la realización de prácticas con profesionales.

No obstante, con el fin de garantizar un servicio de calidad a los ciudadanos, que careciendo de recursos para litigar, solicitan la designación de abogado por turno de oficio, en el Colegio de Abogados de Madrid se acordó exigir a los profesionales que desearan prestar este servicio público, formación adicional y experiencia profesional previa. A tal efecto, se aprobó una normativa interna que regulaba los requisitos exigidos para cursar alta en turno de oficio, adelantándose así el Colegio a las normas que posteriormente se fijarían, ya que esta normativa interna se trasladó en el año 1995 a posteriores normas legales, Real Decreto 108/1995, Real Decreto 2103/1996 y Orden Ministerial de 3 de junio de 1997, en las cuales se fijaban requisitos de formación y experiencia profesional para la prestación del turno de oficio:

a) Tener residencia habitual y despacho abierto en el ámbito del colegio respectivo, así como tener cumplidas todas las obligaciones estatutarias.

b) Acreditar más de tres años en el ejercicio de la profesión.

c) Estar en posesión del diploma del curso de Escuela de Práctica Jurídica o de cursos equivalentes homologados por el Colegio, o, en su caso, haber superado los cursos o pruebas de acceso a los servicios de turno de oficio y asistencia letrada al detenido establecidos por las Junta de Gobierno.

Excepcionalmente, se podrá dispensar motivadamente del cumplimento del requisito establecido en la letra c) si concurren méritosy circunstancias que acreditasen su capacidad. Además de los expresados, para el acceso a los turnos más especializados (Extranjería y refugio, Menores, Jurado, Vigilancia penitenciaria, Constitucional, Violencia de género), se requiere formación adicional y más años de antigüedad en el ejercicio profesional (entre tres y diez años en el caso del Turno Constitucional). Estos requisitos específicos vienen establecidos en las Normas Reguladoras de Turno de Oficio.

\subsection{EI EURO-PSY y el Certificado Europeo de Experto en Psicoterapia de la EFPA ${ }^{7}$}

La creciente internacionalización de la economía y la implantación de un mercado común interno dentro de la Unión Europea ha estimulado la movilidad de los profesionales y la puesta en común de criterios homogéneos de cualificación profesional en los países que la constituyen, de forma que se facilite la libertad en la prestación de servicios $^{8}$. Los psicólogos, como otros profesionales, deben tener la oportunidad de formarse y ejercer su profesión en cualquier lugar de la UE. Los clientes, sean ciudadanos o instituciones, tienen el derecho de elegir servicios de psicólogos competentes de acuerdo con sus intereses y 
derechos en cualquier lugar de la UE. A estos objetivos obedecen las iniciativas EuroPsy, Certificado Europeo de Psicología y el Certificado Europeo de Psicólogo Especialista en Psicoterapia de la European Federation of Psychological Associations (EFPA).

Aunque se debería pretender la uniformidad, transparencia y flexibilidad cuando se pasa de un sistema educativo y profesional a otro cruzando las fronteras nacionales, esos objetivos no son fáciles de lograr, considerando la diversidad de los sistemas y prácticas que se han desarrollado con el tiempo. Hay que encontrar marcos comunes para comparar y establecer la equivalencia de cualificaciones profesionales y educativas, y hay que establecer estándares comunes para garantizar niveles de conocimiento experto y calidad profesional en la UE.

LA EFPA, durante las últimas décadas, ha promovido una serie de iniciativas para establecer un marco y unos estándares comunes. Así, en 1990 aprobó un conjunto de Estándares Óptimos para la Profesión de la Psicología (EFPPA, 1990) en la que se establecieron los requisitos para la educación académica y la formación profesional de los psicólogos. La Red Europea de Psicólogos del Trabajo y de las Organizaciones (ENOP), con una ayuda Copérnico de la Comisión Europea, elaboró un marco curricular y unos estándares mínimos para la psicología organizacional y del trabajo (Roe et al., 1994; ENOP, 1998). Un grupo de trabajo de psicólogos vinculado a la EFPA definieron un marco para la educación y formación de los psicólogos Europeos en el contexto del programa Leonardo da Vinci de la UE (Lunt, 2000; Lunt et al., 2001, Lunt, 2002). El documento resultante, titulado "EuroPsyT: Un marco para la educación y entrenamiento de los Psicólogos en Europa" fue adoptado por la EFPA en 2001. Este marco constituye la base de posteriores desarrollos que han desembocado en un sistema de certificado de dos niveles, que incluirá el EuroPsy (Certificado Europeo en Psicología) y un número de Certificados Europeos Avanzados en Psicología (actualmente sólo está implantado el de Psicoterapia). Un aspecto importante de este trabajo es el desarrollo de competencias que pueden ser evaluadas como resultados de la formación y del entrenamiento, en lugar de basarse únicamente en el currículum académico. La Comisión Europea también está a favor de un acercamiento basado en competencias, que permita la evaluación de la competencia en diversos contextos, tal y como puede comprobarse en el proyecto "Tuning".

\section{EuroPsy, Certificado Europeo en Psicología}

El EuroPsy (Certificado Europeo en Psicología) pretende proporcionar un estándar común de educación académica y de formación profesional de los psicólogos europeos, que informe a los clientes, empleadores y profesionales de que un psicólogo ha adquirido las competencias necesarias para prestar servicios psicológicos en todos los países donde se establezca. El certificado Europsy se concede por la EFPA a aquellos psicólogos que cumplan las condiciones detalladas más adelante, que podrán denominarse "Psicólogos Registrados EuroPsy".

El objetivo del EuroPsy es establecer un estándar que se refiere a la práctica dependiente o independiente, en uno o más contextos profesionales, en el inicio de la profesión. Este estándar define unos requisitos mínimos. El EuroPsy no representa una licencia para la práctica y no pretende sustituir o reemplazar las normativas nacionales de autorización, aunque se espera que sea tenido en cuenta en los futuros cambios que regulen la licencia para el ejercicio de la profesión en cada país. De hecho en España, el EuroPsy ha sido uno de los documentos base para la elaboración del Libro Blanco del Titulo de Grado en Psicología de la ANECA, y los nuevos planes de estudio de las titulaciones de posgrado están siguiendo sus directrices.

El EuroPsy parte de los siguientes principios rectores

1. Promover la disponibilidad de servicios psicológicos adecuados en Europa. Cada ciudadano y cualquier institución debería poder obtener servicios psicológicos de un profesional competente y cualificado, y el sistema debería contribuir a la consecución de este objetivo.

2. Proteger a los consumidores y ciudadanos de Europa a través de una garantía de calidad y proteger al público en general de proveedores de servicios no cualificados.

3. Promover la movilidad de los psicólogos permitiéndoles practicar en cualquier lugar de Europa, siempre que tengan las cualificaciones adecuadas.

4. Asegurar que el EuroPsy se concede sobre la base de: (a) cumplimiento demostrado de un currículum académico en Psicología de suficiente amplitud; (b) competencia demostrada en el desempeño de los ro- 
les profesionales durante la práctica supervisada; (c) acatamiento de los normas éticas europeas (así como nacionales) de los psicólogos.

5. Asegurar que el sistema EuroPsy es equitativo y evita favorecer o desfavorecer a los psicólogos basándose en diferencias nacionales $u$ otras referidas al contexto educacional o profesional, y que reconozca la alta calidad de servicio como un principio fundamental. Esto implica que EuroPsy no plantea requisitos específicos en relación a la estructura o formato de la educación académica, o a la naturaleza y organización de las prácticas preprofesionales.

6. Garantizar la cualificación para la práctica psicológica a nivel de entrada a la profesión así como con posterioridad.

7. Asumir el compromiso de mantener activamente el nivel de competencia profesional. Por esta razón EuroPsy se concede durante un período de tiempo limitado (siete años), y se renueva, también por períodos limitados, basándose para ello en una práctica y un desarrollo profesional continuados.

8. Respetar las normativas nacionales vigentes para los psicólogos.

Marco y estándares mínimos para la educación y entrenamiento de psicólogos.

Sólo los psicólogos que puedan demostrar que han seguido un currículum que cumple los requisitos que describiremos a continuación y hayan realizado una práctica supervisada durante el tiempo equivalente a un año, lo que supone en un total al menos seis años de formación (360 ECTS), pueden optar a obtener el EuroPsy y a ser inscritos en el Registro.

El EuroPsy plantea un modelo de currículum que distingue

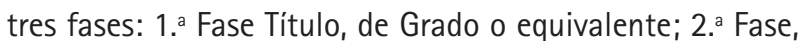
Título de Máster o equivalente; $3 .^{a}$ Fase, Práctica supervi-

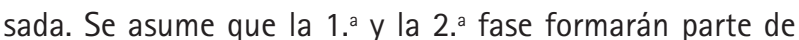
un currículum académico de psicología, mientras que la $3 .^{a}$ fase puede estar incluida o no en el currículum universitario. Se asume también que puede haber una diversidad de opciones del currículum en las universidades que se adapten a estos requisitos, y que no es esencial una estructura de fases separadas o secuenciales. El EuroPsy no obliga a ninguna organización y secuencia del programa de aprendizaje determinada.

Debería señalarse que el Máster o grado equivalente, obtenido tras cinco años de estudio (300 ECTS), se considera que proporciona la cualificación básica necesaria para la práctica de la psicología y ha de estar completada por una práctica supervisada antes de que el individuo sea considerado competente para la práctica independiente. La práctica profesional especializada en cualquier área de la psicología, generalmente requerirá una formación posterior a la cualificación básica en áreas como la Psicoterapia que analizaremos más adelante.

\section{Descripción del contenido del currículum}

\section{Primera Fase}

La primera fase está típicamente dedicada a la orientación de los estudiantes en las diferentes disciplinas psicológicas y también incluye formación en disciplinas relacionadas. Ofrece una educación básica en todas las disciplinas de la psicología, y en las principales teorias y técnicas de la psicología. Proporciona una introducción básica a las habilidades de los psicólogos y una base para la investigación en psicología. No lleva a cualificación ocupacional alguna en psicología y no proporciona la competencia necesaria para la práctica independiente en psicología. Aunque la primera fase puede cubrirse con un programa de tres años conducente a la obtención del Grado (Bachelor), puede también llevarse a cabo en un período mayor, e integrado con el correspondiente a la segunda fase en la que se proporciona el conocimiento, habilidades y comprensión requeridos para la práctica profesional como psicólogo. El currículum de la primera fase está basado en el proyecto "EuroPsyT: Un marco para la educación y el entrenamiento de los psicólogos en Europa". Este marco y los principios generales se presentan en la tabla 1. 
TABLA 1: PRIMERA FASE

\begin{tabular}{|c|c|c|c|}
\hline Tipo de contenido/objetivos & Individuos & Grupos & Sistemas/Sociedad \\
\hline $\begin{array}{l}\text { Orientación } \\
\text { Conocimiento }\end{array}$ & \multicolumn{3}{|c|}{$\begin{array}{l}\text { Métodos en psicología. Historia de la psicología } \\
\text { Visión general de especialidades y campos profesionales en psicología }\end{array}$} \\
\hline $\begin{array}{l}\text { Teorias explicativas } \\
\text { Conocimiento }\end{array}$ & \multicolumn{3}{|c|}{$\begin{array}{l}\text { Psicologia General. Neuropsicología Psicobiología } \\
\text { Psicología Cognitiva. Psicología Diferencial } \\
\text { Psicología Social. Psicología Evolutiva. Psicología de la Personalidad. Psicología del Trabajo y de las } \\
\text { Organizaciones. Psicología Clínica y de la Salud } \\
\text { Psicología Educativa. Psicopatología }\end{array}$} \\
\hline $\begin{array}{l}\text { Teorias tecnológicas } \\
\text { Conocimiento }\end{array}$ & \multicolumn{3}{|c|}{$\begin{array}{l}\text { Teoria de datos y test } \\
\text { Teoría sobre cuestionarios } \\
\text { Teoría sobre evaluación }\end{array}$} \\
\hline $\begin{array}{l}\text { Teorias explicativas } \\
\text { Habilidades }\end{array}$ & \multicolumn{3}{|c|}{$\begin{array}{l}\text { Entrenamiento en habilidades de evaluación } \\
\text { Entrenamiento en habilidades de entrevista }\end{array}$} \\
\hline $\begin{array}{l}\text { Teorias tecnológicas } \\
\text { Habilidades }\end{array}$ & \multicolumn{3}{|c|}{$\begin{array}{l}\text { Entrenamiento en construcción de tests y cuestionarios } \\
\text { Entrenamiento en intervención de grupos }\end{array}$} \\
\hline $\begin{array}{l}\text { Metodología } \\
\text { Conocimiento }\end{array}$ & \multicolumn{3}{|c|}{$\begin{array}{l}\text { Introducción a los métodos: Métodos experimentales } \\
\text { Métodos cualitativos y cuantitativos }\end{array}$} \\
\hline $\begin{array}{l}\text { Metodología } \\
\text { Habilidades }\end{array}$ & \multicolumn{3}{|c|}{$\begin{array}{l}\text { Práctica experimental } \\
\text { Práctica metodológica \&t estadística } \\
\text { Entrenamiento en adquisición de datos y análisis cualitativo }\end{array}$} \\
\hline $\begin{array}{l}\text { Habilidades académicas } \\
\text { Habilidades }\end{array}$ & \multicolumn{3}{|c|}{$\begin{array}{l}\text { Obtención de información de la biblioteca y habilidades bibliográficas } \\
\text { Leer/escribir un trabajo } \\
\text { Ética }\end{array}$} \\
\hline $\begin{array}{l}\text { Teorias no psicológicas } \\
\text { Conocimiento }\end{array}$ & \multicolumn{3}{|c|}{$\begin{array}{l}\text { Epistemología. Filosofía. Sociología } \\
\text { Antropología }\end{array}$} \\
\hline
\end{tabular}

\section{Segunda Fase}

El programa de la segunda fase prepara al estudiante para la práctica profesional independiente como psicólogo. Esta parte del currículum puede ser generalista y preparar para una formación posterior en doctorado o para el empleo como "profesional generalista" en psicología, o ser diferenciada y preparar para la práctica dentro de un área particular profesional de la psicología, como (i) psicología clínica o de salud, (ii) psicología educativa o escolar, y (iii) psicología del trabajo y de las organizaciones.

Como parte de la segunda fase el estudiante, tanto si se prepara para la investigación como si lo hace para el ejercicio como psicólogo profesional, tiene que demostrar la capacidad para realizar la investigación.
La Tabla 2 que se presenta a continuación presenta un modelo de estructura para la segunda fase de formación.

El objetivo del practicum (o "stage" en algunos países europeos) es el de proporcionar un entrenamiento introductorio en el campo profesional. Normalmente se lleva a cabo durante la segunda mitad del currículum universitario, pero puede empezar antes y/o continuarse después de finalizar el currículum. En el último caso, debería ser una responsabilidad conjunta de la universidad y del colegio profesional nacional. La duración debería ser normalmente de al menos tres meses (o 15 ECTS), según el área específica de interés. 
TABLA 2: SEGUNDA FASE

\begin{tabular}{|c|c|}
\hline Tipo de contenido & \\
\hline $\begin{array}{l}\text { Orientación } \\
\text { Conocimiento }\end{array}$ & Orientación sobre el contexto de práctica y las posibilidades de especialización. \\
\hline $\begin{array}{l}\text { Teorias Explicativas } \\
\text { Conocimiento }\end{array}$ & $\begin{array}{l}\text { Cursos en teorias explicativas de psicología general, y/o psicobiología, y/o psicología evolutiva, y/o psicología } \\
\text { de la personalidad, y/o psicología social. E.g. teorías de aprendizaje, teoría cognitiva, teorías avanzadas de la } \\
\text { personalidad. }\end{array}$ \\
\hline & $\begin{array}{l}\text { Cursos en teorias explicativas de psicología del trabajo y de las organizaciones y/o psicología educativa y/o } \\
\text { psicología clínica y/o subdisciplinas psicológicas. E.g. teorias del desempeño laboral, teorias de la cognición } \\
\text { contextualizada, teorías de liderazgo, teorias de desórdenes de personalidad. }\end{array}$ \\
\hline $\begin{array}{l}\text { Teorías Tecnológicas } \\
\text { Conocimiento }\end{array}$ & $\begin{array}{l}\text { Cursos en teorías tecnológicas de psicología general, y/o psicobiología y/o psicología del desarrollo y/o } \\
\text { psicología de la personalidad, y/o psicología social. E.g. Teoria psicométrica, teoria de la evaluación de EEG. }\end{array}$ \\
\hline & $\begin{array}{l}\text { Cursos en teorias tecnológicas de psicología del trabajo y de las organizaciones y/o psicología educativa y/o } \\
\text { psicología clínica y/o subdisciplinas psicológicas. E.g. teorias de análisis del trabajo, análisis de necesidades } \\
\text { de aprendizaje, teorías de asesoramiento y psicoterapia. }\end{array}$ \\
\hline $\begin{array}{l}\text { Teorias Explicativas } \\
\text { Habilidades }\end{array}$ & $\begin{array}{l}\text { Entrenamiento en habilidades para la aplicación de las teorias explicativas arriba mencionadas a la } \\
\text { evaluación en situaciones de investigación/laboratorio. E.g. entrenamiento en registro EMG, entrenamiento } \\
\text { en evaluación de la personalidad. }\end{array}$ \\
\hline & $\begin{array}{l}\text { Entrenamiento en habilidades para la aplicación de las teorías explicativas arriba mencionadas en la } \\
\text { evaluación en situaciones aplicadas y de campo. E.g. entrenamiento en análisis de errores, evaluación de } \\
\text { trastornos de aprendizaje. }\end{array}$ \\
\hline & $\begin{array}{l}\text { Entrenamiento en habilidades para la aplicación de las teorias tecnológicas arriba mencionadas en } \\
\text { intervenciones en situaciones de investigación/laboratorio. P.e. entrenamiento en construcción de test, } \\
\text { diseño de un experimento de aprendizaje. }\end{array}$ \\
\hline & $\begin{array}{l}\text { Entrenamiento en habilidades para la aplicación de las teorias tecnológicas arriba mencionadas en } \\
\text { intervenciones en situaciones aplicadas/de campo. E.g. entrenamiento en el diseño de sistemas de } \\
\text { puntuación de ejecución, diseño de un sistema de entrenamiento, el desarrollo de un plan terapéutico, } \\
\text { psicoterapia. }\end{array}$ \\
\hline $\begin{array}{l}\text { Metodología } \\
\text { Conocimiento }\end{array}$ & $\begin{array}{l}\text { Diseño de Investigación Avanzada } \\
\text { Estadistica multivariada básica y avanzada, incluyendo ANOVA } \\
\text { Análisis de regresión múltiple, análisis factorial } \\
\text { Diseño de Investigación Cualitativa, incluyendo entrevista, uso de cuestionarios y análisis avanzado de datos } \\
\text { cualitativos. }\end{array}$ \\
\hline $\begin{array}{l}\text { Metodología } \\
\text { Habilidades }\end{array}$ & Entrenamiento en habilidades en los métodos y técnicas arriba mencionados. \\
\hline $\begin{array}{l}\text { Habilidades académicas y } \\
\text { profesionales generales } \\
\text { Habilidades }\end{array}$ & $\begin{array}{l}\text { Entrenamiento en habilidades para escribir informes y artículos } \\
\text { Entrenamiento en habilidades de entrevista profesional, etc. }\end{array}$ \\
\hline $\begin{array}{l}\text { Teorias no psicológicas } \\
\text { Conocimiento }\end{array}$ & $\begin{array}{l}\text { Cursos teóricos y prácticos sobre temas de otras disciplinas, relevantes para la actividad profesional. E.g. } \\
\text { medicina, derecho, economía de empresa. }\end{array}$ \\
\hline Competencia básica & INVESTIGACIÓN \\
\hline Competencia básica & PRACTICUM \\
\hline
\end{tabular}


Los estudiantes que finalicen la educación y el entrenamiento completos deben haber desarrollado competencias básicas en habilidades de investigación y habrán llevado a cabo un pequeño proyecto de investigación. Éste puede realizarse en el laboratorio de la universidad o en situación de campo, y puede utilizar enfoques experimentales o más naturalistas como cuasi-experimentos, estudios de caso, entrevistas o estudios con cuestionarios. Los estudiantes serán iniciados en cuestiones concernientes a la naturaleza y ética de la investigación psicológica, y a los métodos básicos empleados por los psicólogos. Esta actividad se llevará a cabo en (esto es 15-30 ECTS).

\section{Tercera Fase}

La tercera fase de la educación profesional de los psicólogos consiste en una práctica supervisada dentro de un área particular de la psicología profesional. Este entrenamiento generalmente tiene lugar después de completar la segunda fase, y a menudo se produce después de dejar la universidad. Sin embargo puede formar parte también de la formación universitaria. Su duración será de 12 meses 0 el equivalente (60 ECTS). Consiste en un trabajo semiindependiente como psicólogo bajo supervisión en un contexto colegial profesional. Esta forma de entrenamiento es considerada esencial para obtener la cualificación profesional de psicólogo, puesto que la aplicación del conocimiento y habilidades adquiridas durante la primera y segunda fase en una situación profesional es un prerrequisito para el desarrollo de las competencias de los psicólogos. Los graduados que han completado la primera y segunda fases sin un período de práctica supervisada no pueden ser considerados cualificados para el trabajo independiente como psicólogo.

Requisitos mínimos del programa de educación y entrenamiento.

El currículum debe tener una duración de al menos cinco años (300 ECTS); Puede ser dividido en 180 unidades para la primera fase y 120 unidades para la segunda fase, aunque las universidades y los países diferirán en la estructura de sus sistemas educativos. La duración de la tercera fase (práctica supervisada) debe ser de al menos un año (60 ECTS) o su equivalente. Esto lleva a una duración total de seis años o 360 ECTS.
El EuroPsy establece también la composición del Currículo estableciendo un mínimo de créditos por áreas, pero como es lógico su descripción sería demasiado prolija para la extensión de este artículo.

\section{Desarrollo Profesional Continuo (DPC)}

El Certificado EuroPsy incluye el concepto de aprendizaje a lo largo de la vida, y exige para su renovación cada siete años que el solicitante haya actualizado sus conocimientos psicológicos. Esto se conseguirá por medio de la experiencia laboral y el desarrollo profesional personal, y mediante el cumplimiento de requisitos del DPC, establecido por el Colegio o Asociación Profesional del país si éstos existiesen. Donde no existan requisitos DPC locales, se proporcionan las siguientes guías para uso de los Comités Nacionales en la renovación del Certificado:

- El solicitante debe aportar evidencia del trabajo profesional como psicólogo que no sea menor de 400 horas por año promediado por un período no menor de cuatro años sobre el período de los últimos seis años antes de la solicitud de renovación.

- Es una responsabilidad del Psicólogo EuroPsy Registrado mantenerse informado acerca de los desarrollos científicos profesionales recientes en psicología, incluyendo, pero no limitándose a su propio contexto de práctica. Se recomienda un mínimo de 80 horas por año y los solicitantes han de aportar evidencia explícita de 40 horas de desarrollo profesional continuo por año. Hay un amplio rango de actividades de desarrollo profesional y la siguiente lista no pretende ser exhaustiva. Para cada tipo de actividad se indica un mínimo y/o máximo aproximado del porcentaje de tiempo total anual que puede ser acreditado con el fin de garantizar la diversidad de actividades para su DPC.

- Asistencia certificada y participación en cursos acreditados y/o talleres dirigidos al desarrollo profesional contínuo (15-60\%)

- Desarrollo de nuevas habilidades específicas a través de la práctica en el trabajo (15-20\%)

- Asistencia certificada a reuniones de supervisión por pares (10-20\%)

- Asistencia certificada a un congreso profesional o científico (10-20\%) 
- (Co-)autoría y/o edición de publicaciones de investigación y/o temas profesionales (max 30\%)

- Presentaciones a audiencias profesionales (max 20\%)

- Trabajo editorial en revistas y libros de psicología (max $20 \%)$

- Para el propósito de la acreditación la suma de las tres categorias finales no puede exceder del $60 \%$.

- Se requiere a los Psicólogos registrados en el EuroPsy que mantengan un registro de sus actividades de Desarrollo Profesional Continuo. Además de registrar la adquisición de experiencia práctica en el contexto de nuevas funciones, grupos de clientes y contextos, el registro debería cubrir entrenamiento y desarrollo de educación continua

\section{Certificado de Acreditación de Psicólogos Especialistas en Psicoterapia}

Dentro del esquema de acreditación de la formación y experiencia profesional de la EFPA, los Certificados Avanzados especifican los requisitos para la práctica independiente en un contexto particular, o de especialización. En el momento actual sólo está en marcha la Acreditación en Psicoterapia a la que ya han accedido más de cuatrocientos psicólogos españoles.

\section{El Certificado de Acreditación para Psicólogos Espe-} cialistas en Psicoterapia pretende ser un instrumento de reconocimiento común en el ámbito europeo entre las diferentes asociaciones y colegios profesionales, que facilite la libre circulación de los psicólogos especialistas en Psicoterapia. La obtención del mismo proporciona al psicólogo el reconocimiento público de su capacitación como psicoterapeuta a través del Comité Internacional de Acreditación de Psicólogos Especialistas en Psicoterapia de la EFPA $y$, en aquellos paises donde haya sido aprobado, como es el caso de España, por el Comité Nacional de Acreditación de Psicólogos Especialistas en Psicoterapia.

La certificación reconoce, de cara a la contratación laboral, que un psicólogo posee las competencias necesarias para ejercer la Psicoterapia como experto, y permite al público identificar a aquellos psicólogos que están cualificados y cuentan con las habilidades necesarias para ser Psicólogos
Especialistas en Psicoterapia. Demuestra, además, que son psicólogos comprometidos con el desarrollo personal y con la formación profesional continuada, y supone una marca de calidad, que no limita el ejercicio profesional de aquellos psicólogos que no deseen solicitarlo o que no cumplan los requisitos en un momento determinado.

A partir de 2012, para obtener el Certificado será preciso realizar programas de formación en psicoterapia acreditados por la EFPA. Sin embargo, en los países donde todavía no existen cursos de especialización en Psicoterapia acreditados por la EFPA (como es el caso de España actualmente), el Comité Internacional de Acreditación de Especialistas en Psicoterapia de la EFPA reconoce la necesidad de evaluar las solicitudes de acreditación de manera flexible. Esto implica tener en cuenta, necesariamente, la diversidad de formación y experiencia con la que van a contar los distintos solicitantes. No obstante, éstos deberán demostrar que poseen el nivel de formación, experiencia y habilidades necesarias para ejercer profesionalmente al nivel de competencia que se exige, y cumplir los siguientes requisitos:

- Haber estado colegiado y haber ejercido la psicoterapia durante al menos seis de los últimos diez años, y al menos tres de los últimos cinco años de forma continua.

- Suscribir la adhesión a los principios éticos,

- Cumplimentar un formulario sobre su visión personal de los principios de la psicoterapia, su formación y experiencia profesional, que será valorada por el Comité Nacional de Acreditación.

- Presentar dos informes de evaluadores o dos cartas de presentación profesional de psicoterapeutas acreditados.

- Declaración del supervisor (opcional)

- Presentar un resumen del currículo vital de acuerdo al formato establecido.

Los Colegios Profesionales sólo tienen sentido si recuperan la función social presente en sus orígenes, si aportan un valor añadido a la sociedad, y se desprendes de corporativismos rancios. En la sociedad del siglo XXI deben convertirse en los garantes de la calidad de los servicios de sus colegiados, empleando fundamentalmente tres estrategias: la apuesta por la formación continua de sus colegiados, el desarrollo de sistemas de acreditación profesional como los descritos en este artículo, y el control deontológico. ¿Seremos capaces de superar este reto? 
1 He querido recoger lo que dice nuestro ordenamiento jurídico sobre lo que "debería" ser un Colegio, y en mi opinión, cómo deben entenderse los mismos en los albores del siglo XXI. El autor es consciente del debate social sobre si los Colegios Profesionales están realmente cumpliendo esta función social o si realmente están simplemente defienden intereses corporativos.

2 Existen algunos Colegios que tienen colegiación voluntaria

3 Véase, por ejemplo, el del Colegio de Diplomados en Enfermería www.codem.es/servicios.

El FOCAD, Programa de Formación Continua a Distancia del Consejo General de Colegios Oficiales de Psicólogos www.cop.es/focad/.

4 Recomendaciones, Segunda Reunión Intergubernamental del Proyecto Regional de Educación, PRELAC II (29 y 30 de marzo de 2007).

5 Muchos Colegios ya disponen de estos servicios para acreditar formación o personas, e incluso el Colegio de Médicos de Madrid tiene un sistema de validación de procedimientos médicos. La Comisión Científica y de Investigación valora actuaciones médicas, pautas profesionales, protocolos clínicos, así como consentimientos informados, quirúrgicos y de investigación, que los colegiados realicen en el sistema público o privado, dentro de las consultas o clínicas. Tras su estudio, entrega el documento que acredita su dictamen positivo como garantía de prestigio profesional, calidad asistencial y herramienta en la defensa de posibles reclamaciones por el ejercicio profesional. Más información en www.cci.qualitasqualitatis.com.
6 EFPA European Federation of Psychologists's Associations.

7 El contenido de este apartado y el siguiente es un resumen de la normativa del EuroPsy incluida en la página WEB del Consejo General de Colegios Oficiales de Psicólogos www.cop.es.

8 Muy pocas profesiones (médico, médico especialista, enfermero generalista, odontólogo, veterinario, matrona, farmacéutico y arquitecto), tienen directivas específicas de la Unión europea que determinan los contenidos mínimos de la formación necesaria para que se homologue el título a nivel europeo. Ver Real Decreto 1837/2008, de 8 de noviembre, que transpone al ordenamiento juridico español la Directiva 2005/36/ CE del Parlamento Europeo sobre reconocimiento de cualificaciones profesionales.

\section{BIBLIOGRAFÍA}

EFPPA (1990): Optimal standards for the training of psychologists, Brussels: EFPPA, booklet no. 3 .

ENOP (1998): European curriculum in work and organizational psychology, Reference model and minimal standards. Paris: ENOP/Maison des Sciences de I'Homme.

Lunt, I. (2000): European project funded by the EU under the Leonardo da Vinci program, European Psychologist, 5(2), 162-164.

Lunt, I., Bartram, D., Döpping, J. Georgas, J., Jern, S., Job, R., Lecuyer, R., Newstead, S., Nieminen, P., Odland, S., Peiró, J. M., Poortinga, Y., Roe, R., Wilpert, B., Herman, E. (2001a): EuroPsyT - a framework for education and training for psychologists 
in Europe, Available from EFPPA, Brussels.

Lunt I. (2002): A Common Framework for the training of psychologists in Europe, European Psychologist, 7(3), 180-191
Roe, R. A., Coetsier, P., Levy-Leboyer, C., Peiró, J. M., Wilpert B. (1994): The teaching of Work and Organizational Psychology in Europe, Towards the development of a Reference Model.
The European Work \& Organizational Psychologist, 4(4), 355-365.

Wilpert B. (2002): Projecting a European Diploma in Psychology, European Psychologist, 7(3), 221-225. 